\title{
Preliminary findings of finger independency and visual force control in children with DCD
}

\author{
MARCIO A. OLIVEIRA \\ School of Public Health, University of Maryland, College Park, MD \\ Correspondence to: Marcio Alves de Oliveira, School of Public Health/ University of Maryland, College Park, MD 20742-2611, USA \\ Email: marcio@umd.edu
}

\section{AT A GLANCE \\ Impairments in manipulative skills often observed in children with DCD during everyday activities may be related to deficits in finger independency and not to difficulties in reducing the number of joint/muscle-level degrees of freedom in order to perform a motor task.}

\section{ACKNOWLEDGEMENTS}

We would like to thank the children and their parents for their time and effort in participating in this study.

PUBLICATION DATA

Received 19 Nov 2014

Accepted 4 Jan 2015

Published 23 Nov 2015

\begin{abstract}
BACKGROUND AND AIM: Finger independency and visual force control were studied in children with DCD.
METHOD: Five children, 9 to 10 years of age, diagnosed with DCD and five age-matched typically developing children were asked to perform two isometric tasks, maximum voluntary force production (MVF) and visual force control (VFC), in seven different finger conditions [Index (I), Middle (M), Ring (R), Little (L) IM, IMR, and IMRL]. For the VFC tasks, the participants were asked to continuously control their finger forces at $20 \%$ of the MVF. To examine finger force independency, maximum voluntary force (MVF), force enslaving (FE) and force sharing (FS) values were computed. To analyze the ability of children with DCD to visually control their finger forces, the following performance measures were calculated: rate of force change, initial overshoot, coefficient of variation (CV), root mean square error (RMSe), and inter-trial variability.

RESULTS: The results from the MVF task showed that children with DCD as compared with TD children (a) produced similar levels of maximum finger force; (b) demonstrated less finger independency; (c) had similar finger-force sharing patterns. In addition, from the VFC task we found (d) larger performance errors in children with DCD; (e) and lower inter-trial consistency as compared to their TD peers.

CONCLUSION: Our preliminary findings suggest that the impairments in manipulative skills often observed in children with DCD during everyday activities may be related to deficits in finger independency. Additionally we found that children with DCD do not have difficulties in reducing the number of joint/muscle-level degrees of freedom in order to achieve a common motor task.

KEYWORDS finger | force control | EEG | DCD | children | enslaving
\end{abstract}

\section{INTRODUCTION}

Precise finger force modulation can be considered a critical factor in everyday activities such as handwriting, typing, tying shoelaces or grasping/holding a glass of water. In order to achieve such manipulative tasks, the central nervous system (CNS) needs to control and coordinate the infinite number of available individual digit-tip contact forces and torques. For example, in keyboard typing, the hand has countless solutions for each individual finger movement and infinite possibilities for combinations among the digits. To type a particular word on the keyboard, the CNS must find a solution to this inherent redundancy in the motor system, ${ }^{1,2}$ for both individual finger actions and as well as synergic interactions among the fingers. ${ }^{3-7}$

Children with developmental coordination disorder (DCD) have been reported to have deficits in manipulative skills which affect their performance in daily and school activities. ${ }^{8,} 9$ Previous studies have shown that children with DCD compared with their typically developing (TD) peers have difficulties in controlling finger force pulses, ${ }^{10-12}$ have excessive grip force generation, ${ }^{13,14}$ and greater variability in finger forces during pinching tasks. ${ }^{15}$ We have previously investigated finger strength in children with DCD and their ability to control digit force/torque. ${ }^{16}$ We used three tasks with increasingly greater kinetic redundancies (index pressing, thumb-index pinching and thumb-index pinching torque) to 
test the hypothesis that the poor manipulative behavior, often observed in children with DCD, results from impairment in controlling a large number of kinetic redundancies. Our results showed that although children with $D C D$ demonstrated the same finger strength in each task as the TD children, they had poor control in the task involving a larger number of kinetic redundancies (i.e., the thumb-index pinch torque task). This suggests that their deficits are not related to a lack of strength, but to difficulties in managing tasks that have many kinetic possibilities (i.e., redundancies). In other words, when the number of fingers required in a task increased, the children with DCD had more difficulties. However, in our previous experiment, the task also changed as the fingers increased (i.e., press, pinch, and torque). To disentangle this finding, the current experiment asks whether or not children with DCD have deficits controlling the kinetic redundancies created by inclusion of multiple fingers in the same task (i.e., an isometric force task).

Humans are incapable of complete independent finger movements and forces. ${ }^{17-19}$ However, the development of certain level of finger independency and individual finger force control is desirable for the successful performance of everyday manipulative tasks. Age-related changes in finger independency have been reported in the literature and, in general, these studies have examined finger interactions using single and multi-finger maximum voluntary force (MVF) production tasks. ${ }^{20-22}$ Research has shown that TD children's finger strength (MVF) increases with age. ${ }^{23}$ We replicated these findings in addition to demonstrating that finger independency also increased with age in TD children. ${ }^{20}$ No studies have previously examined hand digit force independency in children with DCD. The first aim of this study, therefore, was to investigate finger independency in children with DCD. Our previous work ${ }^{20}$ revealed that the proportional contribution of each finger to the overall force produced (i.e., force sharing) did not reveal age-related differences. We suggested that force sharing either develops before the age of 6 or is an intrinsic property of hand neuromusculoskeletal system. ${ }^{20}$ Thus, in addition to finger independency, we investigated force sharing amongst the fingers. If this is an inherent system property then we would expect no differences between children with DCD and their TD counterparts. As our previous study suggested ${ }^{16}$ we also expect that finger strength would be similar between children with DCD and TD children.

A second aim of the study was to investigate the effect of visual guided feedback and kinetic redundancy on force control in children with DCD. While single and multi-finger MVF tasks provide information about (a) force magnitude (strength), (b) hand digit force interactions, and (c) finger independency, a visual guided feedback task permits us to assess (d) the use of sensory feedback, and (e) the effect of kinetic redundancy in children with DCD in continuously regulating their force control. In this study we use the same manual task, under different levels of kinetic redundancy (i.e., different number of fingers). We expect children with DCD to have more difficulties controlling the larger number of kinetic redundancies as was suggested in our previous study (Oliveira et al., 2006). However, we know, from previous studies, that children with DCD do not utilize sensory feedback as well as their TD cohorts and that they need more sensory information than TD children to accomplish a task. ${ }^{24,}{ }^{25}$ Since in this study, increasing the number of fingers provides additional sensory information (i.e., somatosensory feedback) we also expect that supplementary information could help the children with DCD's force control as the motor redundancies increase. 


\section{METHODS}

\section{Participants}

Five children ( 4 boys and 1 girl) age 9-10 years (mean $=9.6$ years; SD $=1.13$ months) diagnosed with DCD and five typically developing (4 boys and 1 girl) age-matched children (mean $=10.4$ years; $S D=.67$ months) participated in this study. The diagnosis of DCD was based on a combination of the Movement Assessment Battery for Children (MABC - $1^{\text {st }}$ edition), the Woodcook-Johnson Revised Cognitive Ability Early Development Scale, and a pediatrician's neurodevelopmental examination including the Physical Neurological Examination for Soft Signs (PANESS). Inclusion criteria were a MABC score at or below the 5th percentile, normal cognitive ability, and an independent DCD diagnosis from the pediatrician's evaluation. Children were recruited through referrals from physicians, therapists, education specialists, website, and local advertisements. Typically developing children had no known learning disabilities, were performing in school at grade level, and had MABC scores at or above 25th percentile. All Participants were righthanded (Edinburgh Handedness Inventory $>90 \%$ ). The right-hand length was measured from the middle finger tip to the lunate of the wrist. The width was measured between the metacarpophalangeal joints of the index and little finger. A parent or legal guardian of each child gave his/her informed consent based on the procedures approved by the University of Maryland's Internal Review Board (IRB). A summary of the Participants scores on the MABC and anthropometrics are presented in Table 1.

Table 1 -Description of individual data for sex, age, Movement ABC scores [manual dexterity (MD), ball skills (BS) balance (BL), total score (Tot.) and percentile (\%ile)], body measures for the hand length and width and body height and weight. On the button row of each group, averaged groups of each measure are shown with standard deviation.

\begin{tabular}{|c|c|c|c|c|c|c|c|c|c|c|c|c|c|}
\hline \multirow[t]{2}{*}{ Group } & \multirow{2}{*}{$\begin{array}{l}\text { Subj. } \\
\text { Study } \\
\text { id }\end{array}$} & \multirow[b]{2}{*}{ Grant id\# } & \multicolumn{5}{|c|}{ MABC scores } & \multirow[b]{2}{*}{ Tot. } & \multirow[b]{2}{*}{$\%$ ile } & \multicolumn{3}{|c|}{ Body measures $(\mathrm{cm} / \mathrm{kg})$} & \multirow[b]{2}{*}{$\begin{array}{l}\text { Body } \\
\text { Weight }\end{array}$} \\
\hline & & & Sex & Age & MD & BS & $B L$ & & & $\begin{array}{l}\text { Hand } \\
\text { Length }\end{array}$ & $\begin{array}{l}\text { Hand } \\
\text { Width }\end{array}$ & $\begin{array}{l}\text { Body } \\
\text { Height }\end{array}$ & \\
\hline \multirow{6}{*}{ DCD } & 1 & 06 & $M$ & 8.3 & 14.5 & 10 & 5 & 29.5 & $<1$ & 13.5 & 7.5 & 127.0 & 28.3 \\
\hline & 7 & 17 & M & 8.5 & 8 & 5 & 10 & 23 & $<1$ & 14 & 5.5 & 129.5 & 22.2 \\
\hline & 4 & 10 & M & 10.0 & 15 & 10 & 10.5 & 35.5 & $<1$ & 16 & 6.5 & 139.7 & 26.8 \\
\hline & 2 & 40 & $\mathrm{~F}$ & 10.3 & 11 & 5 & 8.5 & 24.5 & $<1$ & 11 & 7 & 128.3 & 26.1 \\
\hline & 3 & 45 & $M$ & 10.9 & 15 & 10 & 11 & 36 & $<1$ & 15.5 & 6.5 & 147.3 & 37.6 \\
\hline & \multicolumn{2}{|c|}{ Average (SD) } & & $9.6(1.1)$ & $12.7(3.1)$ & $8(2.7)$ & $9(2.4)$ & $29.7(6.0)$ & $<1$ & $14(1.9)$ & $6.6(0.7)$ & $134.4(8.8)$ & $28.2(5.7)$ \\
\hline \multirow{6}{*}{ TD } & 16 & & $M$ & 8.2 & 7.5 & 0 & 0 & 7.5 & 26 & 13.2 & 5.9 & 137.4 & 21.3 \\
\hline & 13 & & $\mathrm{~F}$ & 8.4 & 1 & 5 & 1 & 6 & 36 & 13.5 & 5.5 & 134.6 & 25.3 \\
\hline & 12 & & M & 10.1 & 0 & 5 & 3 & 8 & 22 & 14.5 & 6.5 & 143.6 & 32.5 \\
\hline & 14 & & M & 10.6 & 0 & 3 & 0 & 3 & 65 & 11.2 & 7.1 & 124.5 & 28.1 \\
\hline & 15 & & $\mathrm{M}$ & 10.7 & 0 & 0 & 3.5 & 3.5 & 60 & 18.6 & 7.8 & 128.3 & 36.9 \\
\hline & \multicolumn{2}{|c|}{ Average (SD) } & & $10.4(0.7)$ & $1.7(3.2)$ & $2.6(2.5)$ & $1.5(1.6)$ & $5.6(2.3)$ & $41.8(19.6)$ & $14.2(2.7)$ & $6.6(0.9)$ & $133.7(7.5)$ & $28.8(6.1)$ \\
\hline
\end{tabular}

\section{Apparatus}

To measure force applied by the fingers, four sensors (one for each finger $2^{\text {nd }}-5^{\text {th }}$ digits) with amplifiers (Models 208 M182 and 484B, Piezotronics, Inc.) were used. The sensors were attached on a customized aluminum structure with four slits adjustable according to the hand and finger sizes of the Participants. For detailed description and illustration of the experimental set-up see Shim, et al. (2006). The signals from the sensors 
were conditioned, amplified, and digitized at $100 \mathrm{~Hz}$ with a 16-bit A/D board (PCI 6034E, National Instruments Corp.) with a custom software program made in LabVIEW (LabVIEW 7.1, National Instruments Corp.). A desktop computer (Dimension 4700, Dell Inc.) was used for data acquisition. MatLab (MatLAB 7, MathWorks, Inc.) programs were written for data processing and analysis.

\section{Procedures}

During the experiment, the children sat on a chair facing the computer screen with their shoulders abducted $35^{\circ}$ in the frontal plane and elbows flexed $45^{\circ}$ in the sagittal plane. Their forearms rested in a customized wrist-forearm brace consisting of a piece of foam attached to a semi-circular plastic cylinder fixed to a wooden panel $(29.8 \times 8.8 \times 3.6 \mathrm{~cm})$. Velcro straps were used to reduce forearm and wrist movements.

The children were asked to place each of their distal phalanges in a thimble such that all joints were slightly flexed and formed a dome shape with the hand. The children performed two isometric tasks: maximum voluntary force production (MVF) and visual force control (VFC). In the MVF task the children performed a total of seven conditions: four with a single-finger [Index (I), Middle (M), Ring (R), and Little (L)] and three with multiple fingers (IM, IMR and IMRL). One trial was performed in each condition. During each trial, all fingers were on the thimbles and the children were asked to produce maximum isometric force in flexion over a 3-s interval. The children were instructed to concentrate on the task finger and not on the non-task fingers. At the beginning of each trial, the computer generated a 'get ready' sound and force feedback of the task finger(s) was graphically displayed on the computer screen.

In the VFC task, the children performed an isometric, constant and continuous force at $20 \%$ of his/her maximum force as measured in the MVF task/condition. The children performed a total of four conditions: one for single-finger [Index (I)] and three for multi-finger (IM, IMR and IMRL). A computer screen showed the required force template (dashed and straight line) corresponding to $20 \%$ of the total force (MVF) produced by the child for each finger condition, and the moving cursor which represented the real-time force they were producing. At the start of each trial, the computer generated a voice saying 'get ready' and the Participants were asked to control their finger forces moving the cursor throughout the target force template over $10 \mathrm{~s}$ (Figure 1). Twelve trials of the VFC task were collected, for each finger condition (I, IM, IMR, IMRL) with 30-s rest between trials and five minutes rest between the conditions. The order of the conditions (I, M, R, L, IM, IMR, IMRL) in both tasks (MVF and VFC) was randomized and balanced across participants.

\section{Data Analysis}

For each trial of the MVF task, the force data were all digitally low-pass filtered with a $2^{\text {nd-order, }}$ zero-lag Butterworth filter a $25 \mathrm{~Hz}$ cutoff frequency.26, 27 The instantaneous peak force produced by each finger was measured at the time of maximum force. From these data, the measures of maximum voluntary force (MVF), force enslaving (FE) and force sharing (FS) were calculated. MVF was defined as the maximum force produced by the task finger(s). FE, an index of finger inter-dependency, was calculated as the average of the non-task finger forces. These values were averaged across all fingers

\begin{tabular}{l|l|l|l|ll}
\hline Oliveira & 2015 & VOL. 9 & N.2 & 4 of 19
\end{tabular}


to obtain the overall finger inter-dependency indices FE (Eq. 1)., where $i \neq j, n=4$, where $F_{\max }^{i}$ is the maximum force produced by the finger, $i$, and $F^{i j}$ is the force produced by the non-task finger,$i$, during the $j$ finger maximum force task. ${ }^{21,22,28}$

$$
F E=\sum_{j=1}^{n}\left[100 \% \times \sum_{i=1}^{n}\left(F^{i j} / F_{\max }^{i}\right) /(n-1)\right] / n
$$

Note that $F_{E N}^{j}$ represents the averaged, normalized force of non-task fingers for the same trial. FE for each finger represents the averaged percentage force of non-task fingers for the same trial with respect to the task finger MVF. ${ }^{20} \mathrm{FS}$ of each finger was calculated as the percentage contribution of each finger force to the sum of the finger forces during the four-finger task.

The first trial (in each condition) of the VFC task served as a familiarization trial and was not included in the analyses. For each trial, the time series data analyses were analyzed and the following four performance variables were extracted. (a) Rate of force increasing (Newton/second) was calculated from the start point of the VFC task to the time that the subject's reached the target. (b) Initial force overshoot was computed as an initial performance error of the VFC task. The initial overshoot was defined by the percent value exceeding the $20 \%$ of MVF target force level..$^{29}$ (c) Intra-trial force variability was measured by the coefficient of variation ( $\mathrm{CV}=$ standard deviation/mean) and considered as a performance error based upon the mean force applied by each subject. (d) Intra-trial accuracy was measured by the root mean square error (RMSe) calculated as a performance error based upon the target force ( $20 \%$ of the MVF) of each subject. The time series of VFC trial lasted 15 seconds and is depicted in Figure 1. The trial's start point (T1) was defined based on the average amount of the data at the baseline plus three times the standard deviation of the baseline. Rate of force increasing was calculated from the start point (T1) to the target (T2). Initial overshoot was computed from the target to first maximum peak value recorded beyond the target force level (point T3). CV and RMSe were computed during the constant isometric force control phase, which was defined as five seconds after the first time that the subject crosses the target (T2), between T4 and T5 in the Figure 1. The four initial seconds of the constant phase of the task (T2 to T4) were removed to exclude the initial period of force adjustment to the visual feedback. 


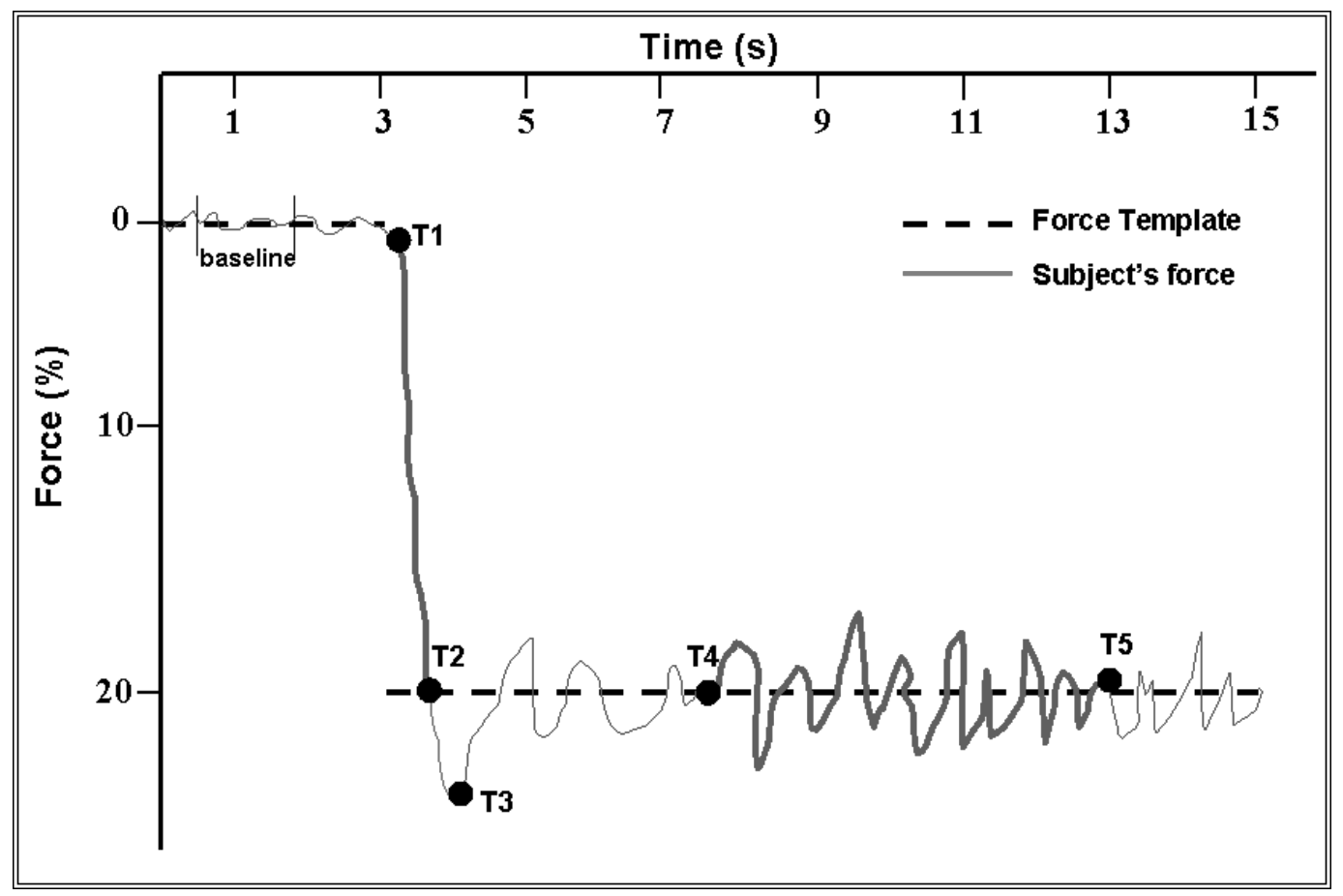

Figure 1. Illustration of the visual feedback provided to the participants on the computer screen during the visual force (VFC) task. The sample contains the force template (dashed lines) and the constant finger force (full line) applied by the participants at $20 \%$ of their maximum voluntary force (MVF).

\section{RESULTS}

\section{Maximum voluntary force production tasks (MVF)}

MVF values showed no significant differences between children with DCD and typically developing children for all finger conditions (I, M, R, L, IM, IMR, IMRL). For a single-finger condition, the index and middle fingers showed relatively large values of MVF while the ring and little fingers showed smaller values (Figure 2). As expected, MVF values became larger as the number of the fingers increased $(|<| M<|M R<| M R L)$. These results were supported by repeated-measures ANOVA for FINGERS, which showed main effect for the factor FINGERS $[F(3,24)=90.6, p<.001]$. The post-hoc analyses showed differences among all finger conditions $(p<.001)$. No GROUP effect or interaction between factors was found. 


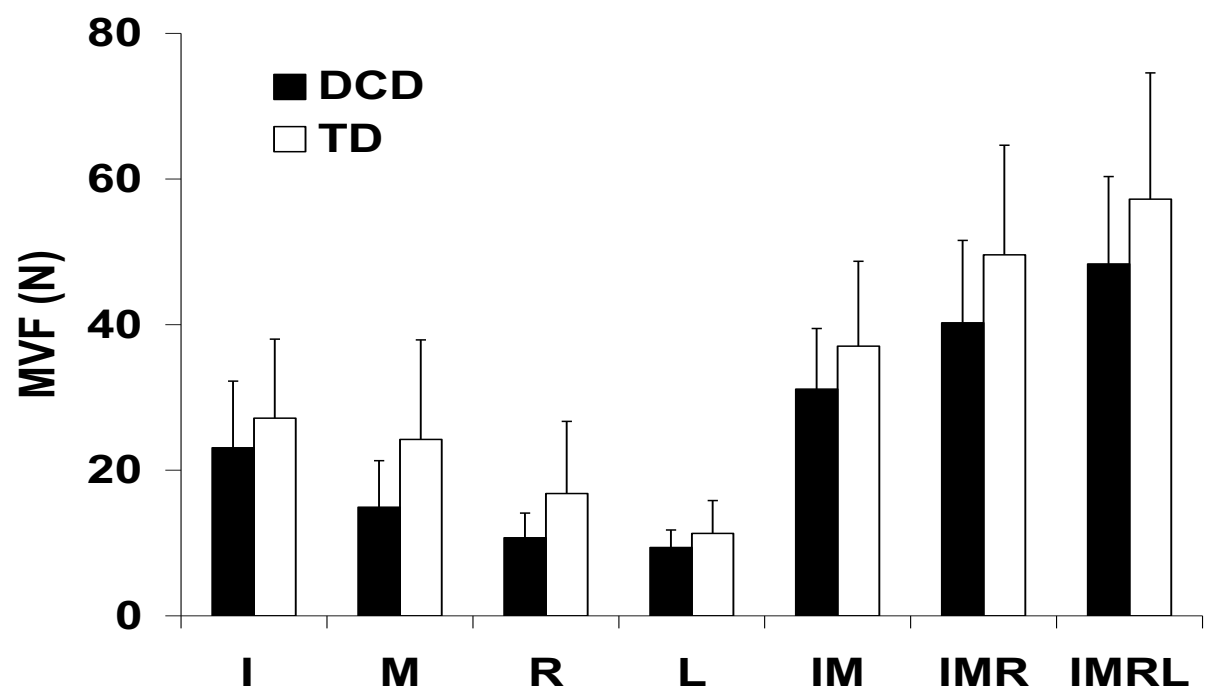

Figure 2: Maximum Voluntary Force (MVF) of index (I), middle (M), ring (R), little (L), index/middle (IM), index/middle/ring (IMR), and index/middle/ring/little (IMRL) fingers during the MVF force production tasks. Averaged group data are shown with standard deviation bars.

While the participants were performing the single-finger MVF production tasks (I, $M, R, L$ ), significant level of force with the uninstructed (non-task) finger forces were also recorded. The forces applied by the non-task finger forces were quantified as a normalized value of its own maximum force (Eq.1) and it was considered as an index of finger independency (FE) such that a lower FE represents greater finger independence. Over all fingers, children with DCD showed larger FE as compared with TD children (Figure 3a). In both groups, the ring and little fingers showed larger FE as compared to index and middle fingers (Figure $3 \mathrm{~b}$ ). These results were supported by the repeated-measures ANOVA which showed significant effects of GROUP $[F(1,8)=10.5, \quad p<.005]$, FINGERS $[F(3,24)=19.5, p<.001]$ and interaction between both factors $[F(1,24)=3.22, p<.005]$.

During the four-finger tasks (IMRL), the participants were asked to produce forces with all four fingers. FS of individual finger forces was calculated as the percentage of each finger force with respect to the total of the four-finger force. FS values showed no significant differences between groups. Overall, the FS of the little finger during the fourfinger task was smaller (13\%) compared to the index (32\%), middle (32\%) and ring (21\%). These findings were supported by the repeated measures ANOVA which showed main FINGERS effect $[F(3,24)=9.96, p<.001]$. Post hoc analyses showed that the contribution of the $L$ finger was smaller than I ( $p=.001), M(p=.015), R(p=.015)$. No GROUP effect or interaction between the factors. 


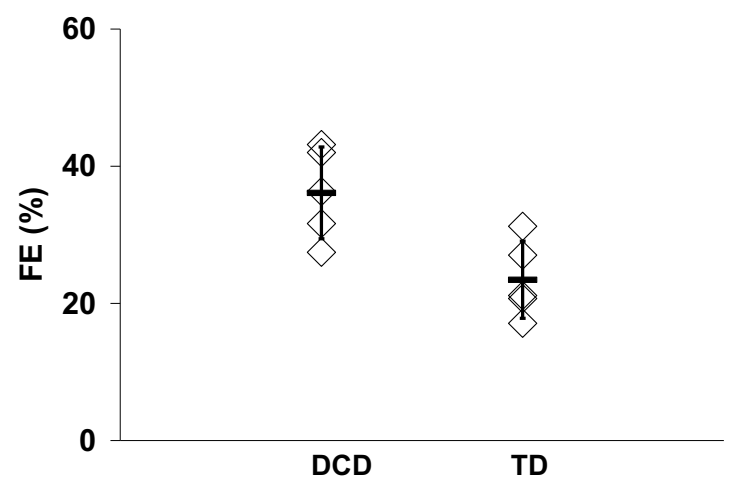

(a)

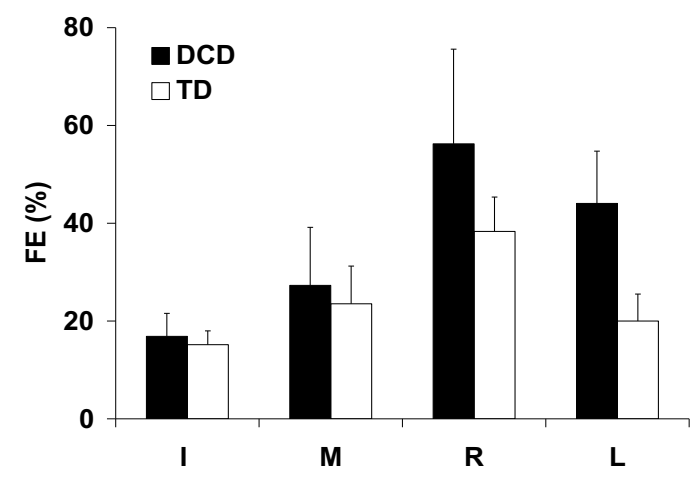

(b)

Figure 3: (a) Force enslaving (FE) values for children with $\mathrm{DCD}$ and TD children. Opened diamonds represent individual data and horizontal traces with standard deviation bars, represent the averaged group data. (b) Groups average of individual finger force enslaving (FE) values (I. M, R, L) with standard deviation bars.

\section{Visual force control task (VFC)}

There was a noticeable difference between children with DCD as compared with TD children on the VFC task profiles (Figure 4). In order to characterize the children's performance during the VFC tasks the slope-line (force applied by the subject towards the target) was first examined. In general, children with DCD were slower and tended to overshoot the task target. Secondly, the constant-line (force constant and continuously applied by the participants to sustain at the target) was analyzed. Performance errors variables were computed to account for the children's visual force control. As observable in the Figure 4, children with DCD were more variable, demonstrating greater overshoot at the start of the trial, and less accuracy in staying at $20 \%$ of their maximum compared to TD children. 


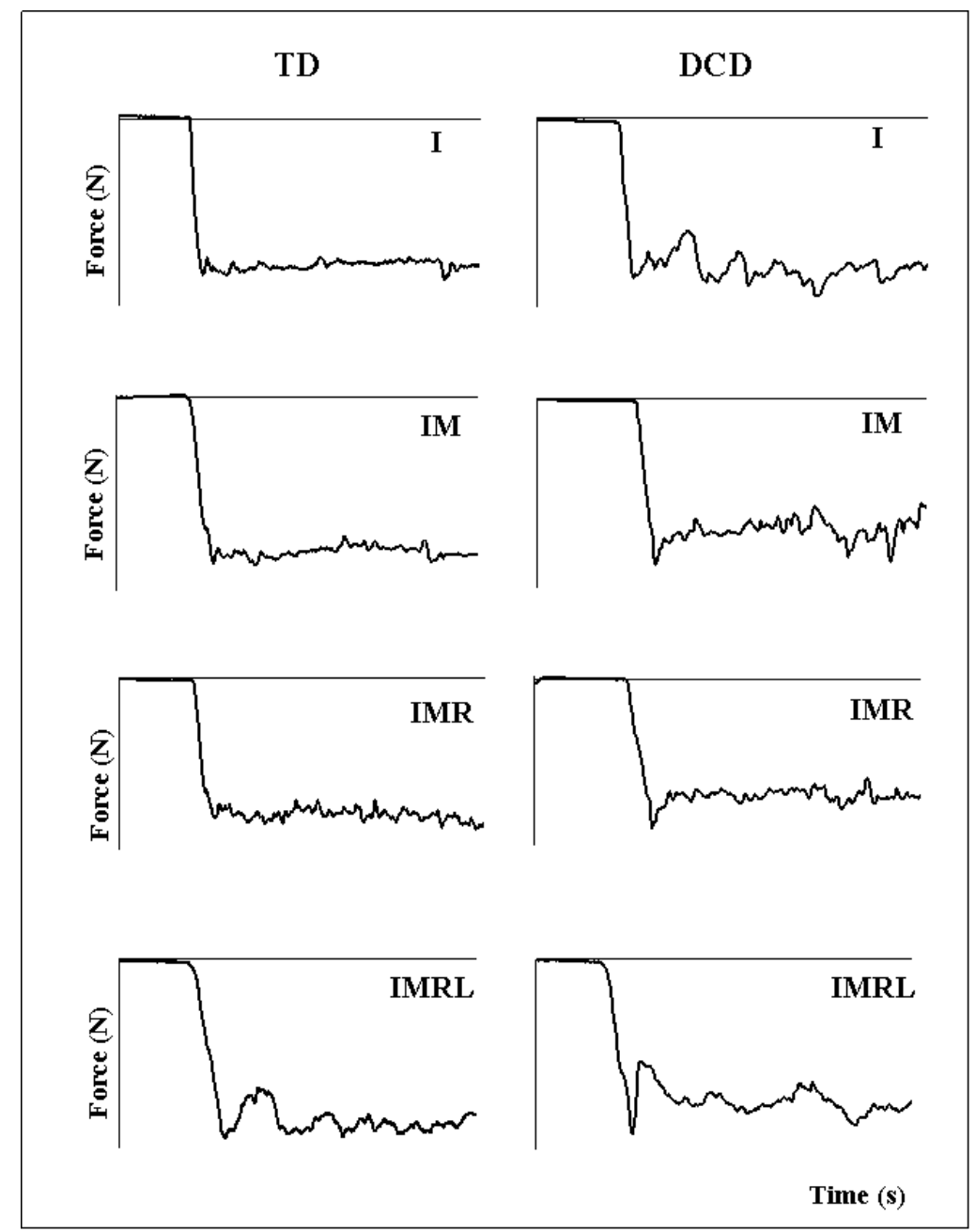

Figure 4: Visual force control (VFC) task. A representative trial of a child with DCD (\#3) and one TD child (\# 9) in each condition (I) index, (IM), index/middle/ring (IMR), and index/middle/ring/little (IMRL) fingers.

The children's rate of force, as the cursor moved to the $20 \%$ target was computed. Children with DCD were significantly slower than TD children. The comparison among the fingers condition indicated that as the number of fingers were added, both groups tended to increase the rate of force (Figure 5a). These results were confirmed by the repeated measure ANOVA which revealed a main effect for the factors $\operatorname{GROUP}[F(1,8)=6.3, p<.001]$ and FINGERS $[F(3,24)=77.0, p<.001]$. No interaction between factors was found. Post hoc tests showed that the rate of force was larger for IMRL as compared with all the others finger conditions $(p<.001)$.

As an initial performance error, we measured the excessive force applied by the subject when they first try to reach the targeted force (20\% of the MVF). As shown in Figure $5 b$, the initial force overshoot values were larger in children with DCD as compared to TD children. Both groups showed greater initial force overshoot during the four-finger (IMRL) condition as compared with the others fingers conditions. These findings were supported by the repeated measures ANOVA, which revealed a significant main effect for GROUPS $[F(1,8)=7.5, p<.001]$ and FINGERS $[F(3,24)=17.1, p<.001]$. No interaction 
between the factors was found. Post hoc tests indicated that the overshooting values were greater during IMRL as compared with I ( $p<.044)$; IM ( $<<.011)$; and IMR $(p<.004)$.

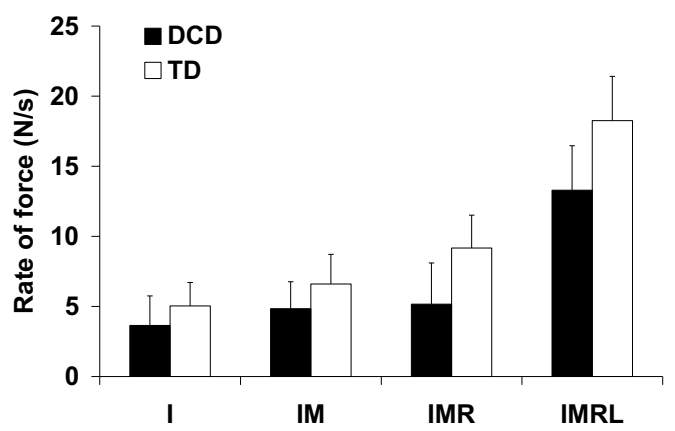

(a)

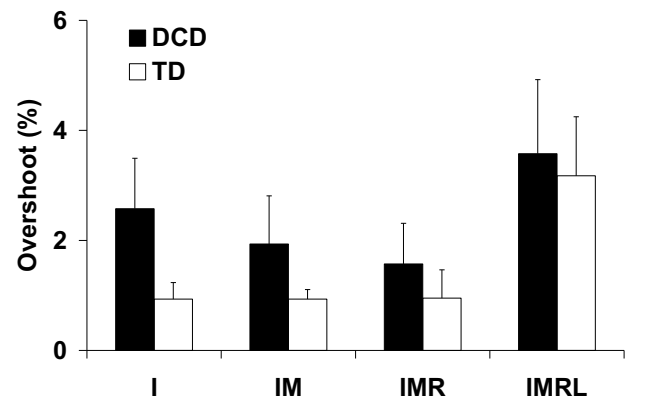

(b)

Figure 5: (a) Rate of force group averaged values (I, IM, IMR, IMRL) for children with DCD and TD children. (b) Initial force overshoot group averaged values (I, IM, IMR, IMRL) for children with DCD and TD children. Averaged groups data are shown with standard deviation bars.

In the VFC task, the children were asked to sustain their finger forces at $20 \%$ of their MVF. The mean values for the VFC task did not differ for the two groups [DCD (19.6\%) and TD (19.95) or among the four finger conditions [I (19.6\%), IM (19.5\%), IMR $(19.5 \%)$, IMRL (19.6\%). These results were supported by the repeated measures ANOVA that showed no main effect for the factors GROUP and FINGERS. Such similarities between group and finger conditions indicate that all participants were able to achieve the task goal (i.e., continuously maintaining the isometric finger forces, on average, around $20 \%$ of the MVF) across all finger conditions.

However, the VFC task did reveal group differences on CV and RMSe values. Children with DCD showed greater CV compared to TD children during the constant phase. There were no differences among the fingers (Figure 6a). These results were supported by the repeated measures ANOVA which showed a main GROUP effect $[F(1,8)=22.7$, $p<.001]$ and no effect for the factor FINGERS or an interaction between factors. The averaged group RMSe values are shown in the Figure 6b. As illustrated, children with DCD presented significantly greater RMSe values as compared to TD children and the RMSe was larger in the I finger task than the IMR and the IMRL tasks. The repeated-measures ANOVA revealed a main effect for the factor GROUP $[F(1,8)=37.2, p<.001]$, and FINGERS $[F(3,24=8.2, p<.001]$. The I finger condition showed greater error between groups as 
compared to their differences in the IMR and IMRL finger conditions. This finding was confirmed by the interaction between GROUP and FINGERS $[F(3,24)=7.4, p<.001]$ found between the factors (GROUP and FINGERS).

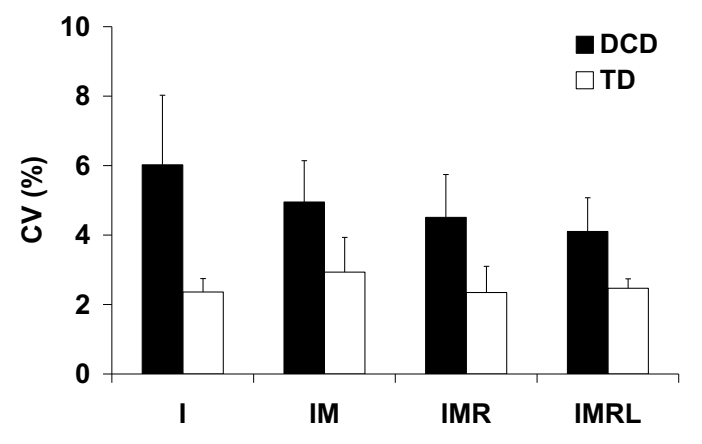

(a)

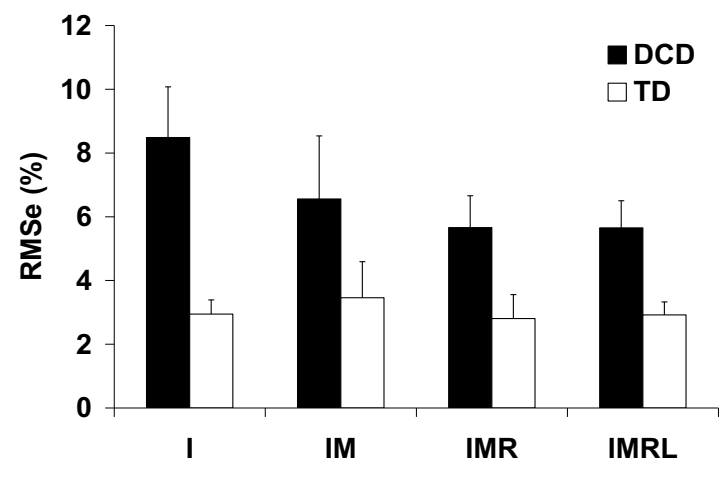

(b)

Figure 6: (a) Coefficient of variation (CV) groups averaged values (I, IM, IMR, IMRL) for children with DCD and TD children. (b) Groups average of individual RMSe values (I, IM, IMR, IMRL). Averaged groups data are shown with standard deviation bars.

The standard deviation of the mean values of the VFC tasks across the twelve consecutive trials performed by each subject was computed in order to characterize intertrial variability (performance consistency). The regression analyses did not show a significant relationship between the children's trials' performance for any finger force condition (I, IM, IMR, IMRL; p>.05). However, on average, children with DCD were significantly more variable (less consistent) across the twelve trials as compared to TD children (Figure 7). No differences among the finger conditions were found. This result was supported by the repeated measures ANOVA which showed main GROUP effect $[F(1,8)=26.7, p<.001]$, no FINGER effect and no interaction. 


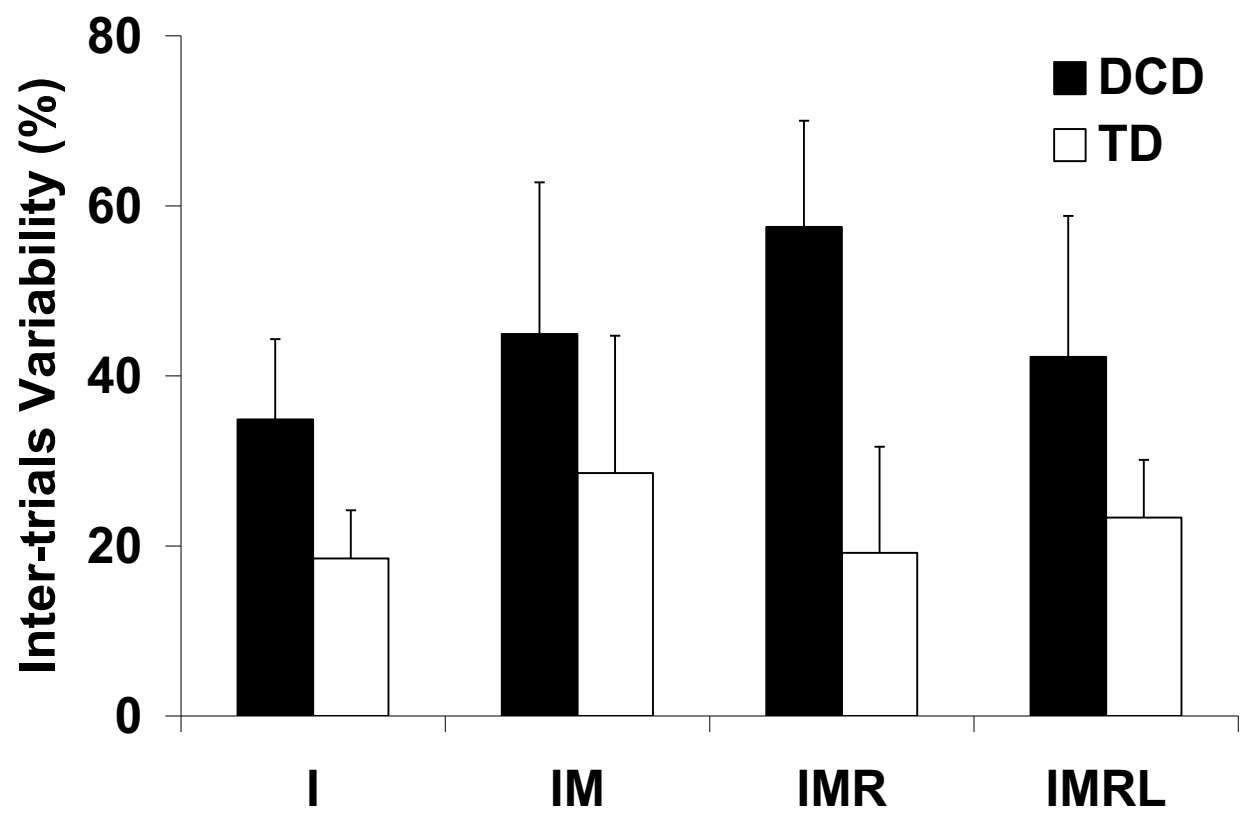

Figure 7: Inter-trial variability values (I, IM, IMR, IMRL) for children with DCD and TD children. Averaged group data are shown with standard deviation bars.

\section{DISCUSSION}

This study investigated finger independency during single-finger and multi-finger maximum voluntary force production tasks in children with DCD and those who are typically developing. In addition, we examined the effects of kinetic redundancy in a visual force control task. Overall, children with DCD showed similar capability as their TD cohorts for maximum finger force production, but revealed impairments in finger independency and visual force control. The individual fingers contributed differently to the total force during the four-finger task, however, these proportions were similar between children with DCD and TD children. During the visual force task, children with DCD, in general, presented greater performances errors and less consistency as compared to the TD children. No performances differences between children with DCD and TD children were found when the number of kinetic redundancies was manipulated.

\section{Maximum force production and finger interaction indices}

The similarity in maximum finger force production between children with DCD and TD children found in this study complements our previous findings ${ }^{16}$ and confirms our first hypothesis that finger strength is similar between children with DCD and TD children. The MVF values found in both groups correspond to those reported by earlier studies in TD children in 9- to 10-year-olds. ${ }^{20,23}$ It has been reported, for example, that the index finger MVF value in children at a similar age-band is about $21.7 \pm 3.9 \mathrm{~N}^{23}$ and $24.7 \pm 4.8 \mathrm{~N} .{ }^{20}$ In the current study, corresponding values were also found for the index MVF production in both groups $(D C D=23.09 \pm 9.15 \mathrm{~N}$; TD $=27.16 \pm 10.85 \mathrm{~N})$. Similar to the index finger MVF 
values, the maximum force values of the others fingers (M, R and $L$ ) were also similar to those previously reported. ${ }^{20}$ Even though everyday manipulative skills do not often require levels maximum of finger force production, these findings question the hypothesis that muscle weakness is a major problem for children with DCD, as well as confirms the premise that the difficulties in multi-digit dexterity, regularly observed in children with $D C D$, is not necessarily related to the magnitude of force itself.

Secondly, we hypothesized that impairment in everyday manipulative tasks in children with DCD may partially result from a lower level of finger independency than their TD age cohorts. The results of the current study showed higher finger enslaving index (FE) in children with DCD as compared with their TD peers, confirming our hypothesis that they would have lower finger independency. Previous studies have suggested that finger independency is critical in everyday manipulation tasks. ${ }^{21,} 22,28,30$ This is the first study to investigate finger independency in children with DCD so there are no comparison data available. The only equivalent result is the FE values for our TD children $(24 \%)$ which were very close to the FE value (26\%) averaged across the 10-year-old children reported in our earlier developmental study. ${ }^{20} \mathrm{FE}$ is an established index of finger independency and its values are inversely proportional to the concept of dependency. A greater level of FE is synonymous with larger finger inter-dependency, which means lower level of independency. Finger independency is critical to finger movement control. $5,18,20$ In order to control a particular finger, the CNS needs to send supplementary commands to the nonintended finger muscles so as not to cause unintended forces during the action. Finger dependency has been explained as influenced by central and peripheral factors. Central factors include the overlapping digit representation in the hand area of the primary motor cortex (M1), ${ }^{19}$ as well as the output divergence from the neurons in the primary motor cortex to innervate the spinal motor neuron pools of different finger muscles. ${ }^{5}$ Peripheral factors consist of biomechanical inter-connections of the soft tissues such as interconnections of the finger tendons, and the insertion of one muscle (ex. Flexor digitorum profundus) to multiple fingers. ${ }^{31}, 32$ Since it has been shown that finger independency increases with age ${ }^{20}$ the smaller level of finger independency found in children with DCD could reflect differences or delays in the development of such peripheral and/or central factors.

While finger independency increases with age the proportional contribution of the individual fingers to the overall force created by the fingers, force sharing (FS), remains constant from the age of six years onwards. ${ }^{20}$ As we hypothesized, there were no differences in the finger FS patterns between the children with DCD and TD children. The current results showed that when performing the four-finger MVF task, children with DCD (I: $37 \%$, M: $28 \%$, R: 20\%, L: 15\%) demonstrate similar interaction among the fingers as compared with their TD peers (I: $35 \%, \mathrm{M}: 26 \%, \mathrm{R}: 24 \%, \mathrm{~L}: 15 \%)$. The results of both groups are very similar to the FS values (I: $30 \%$, M: 33\%, R: 22\%, L: $15 \%$ ) reported by previous studies on children, ${ }^{20}$ as well as those FS values (I: $30 \%, \mathrm{M}: 30 \%, \mathrm{R}: 25 \%$, L: $15 \%$ ) reported for adults. ${ }^{22}$ The present findings for children with DCD lend further support to our earlier speculation that FS as an inherent property of the hand's neuromuscular system or to the possibility that this attribute develops before the age of six. ${ }^{20}$

\section{Visual force control performance}

The VFC results of the initial slope of the cursor to meet the $20 \%$ target showed that children with DCD moved the force cursor slower $(3.03 \mathrm{~N} / \mathrm{s})$ than TD children. Both 
groups responded to the VFC task moving the force cursor beyond the target (i.e., overshooting). However, our results showed that over all tasks, children with DCD showed greater overshooting $(2.4 \% \pm 1.4)$ as compared with TD children $(0.9 \% \pm 0.5)$. The slower movement and increased force overshooting observed in children with DCD suggest difficulty in using a feedforward strategy for predicting the level of the force required by the task. Children with DCD, like young children, rely predominantly upon visual feedback for their visual motor control rather than using integrated feedback and feedforward processing. ${ }^{23,33}$ Developmental studies have shown that as children increase in age they use more effectively a feedforward strategy to predict the target and to control when to stop the force acceleration. ${ }^{23,} 29,34,35$ It has been reported that children around five years of age are able to decrease dramatically their initial force overshoot and tend to undershoot the signal in a similar way to adult's performance, ${ }^{35}$ The overshoot values found in the current study, for both groups were significantly larger from those previously reported in younger children $(5$ years old $=0.3 \%) .{ }^{29}$ Such disparity may be explained by the differences in the experimental tasks. Potter et al., ${ }^{29}$ similarly to many other developmental studies $^{16,23,34-40}$ have asked younger children to press the sensor (thumb/index pinching movements) in order to move the force cursor/line vertically to the target. In these studies, the visual force relationship is familiar and spatially compatible (the harder they pressed the sensors, the higher the cursor moved on the monitor) to the many tasks in everyday activities. By the age of five, children may well have had enough experience with this type of visual force relationship in the "real world". Observing baby toys and computer games, for example, when children push the keys (wrist/fingers flexion) they usually make objects or characters on the screen move/jump up vertically. In our study, we provided an isodirectional task (the sensor was activated by isometric finger flexion downward and the cursor also moved downward on the monitor). Thus, in our study, the visual force relationship was incompatible (the harder they pushed the sensor, the more negatively/downwards the cursor moved to the target level). In future studies, we suggest that the visual force compatibility be systematically manipulated in order to evaluate the effects on finger force estimation including overshooting.

We also evaluated performance errors during the constant force control phase. Force variability (CV) and accuracy (RMSe) were computed to measure the ability of children with DCD to adjust their finger forces to match the required visually-presented level of force displayed on the monitor. In the present study, all children were able to complete the task, however, the children with DCD showed larger finger force variability and less accuracy as compared to the TD children. Even though greater variability and lower accuracy is a main findings from previous studies on finger force control in children with $D C D,{ }^{10-16}$ the results of our current study are the only findings to probe how children with $D C D$ use continuous visual information to regulate their force performance. In previous studies, the visual feedback provided to the children was related to the instant peak force pulses ${ }^{10-12}$ or to a discrepancy between two horizontal lines displayed on the screen. ${ }^{16}$ In these studies, children had no access to the real time and history of the visual tracing of the performance during the finger force task. Indeed, in the previous studies, "memory" may have played an indirect role in the task performance. In our current study, we explored a continuous visual force task. Similarly to Lazarus et al. (1995), we asked the children to sustain their finger forces on the line while the cursor traveled across the screen (by pressing the force sensors). The crucial difference between our current study and the previous ones is that in our VFC task, the children's performance and error 
corrections were based on a visual feedback that provided a time line of their force performance throughout the trial. We intentionally used this type of task in order to provide more visual feedback to the children. Developmental studies have shown that force variability decreases across age due primarily to the increasing ability to use visual feedback ${ }^{36,37}$ and feedforward strategies ${ }^{39}$ efficiently. By providing visual feedback continuously and with a history of the cursor movements, we decreased the memory component of the task or possibly the importance of an internal representation or estimation of $20 \%$ force. Evidence that this feedback presentation made a difference can be found in the difference between the CV values found in our TD children's group (2.4\%) and those previously reported in TD children $(\sim 5 \%)$ at the same age band, ${ }^{23}$ where the participants were asked to match and keep the cursor as steadily as possible over the position of the target.

Motor redundancy has been considered a "problem" to be solved by the CNS in order to control movements ${ }^{1,2}$ and has been extensively explored in human finger force control. ${ }^{3}, 5,7,26,41-44$ Based on this literature and on our previous findings, ${ }^{16}$ we hypothesized that the performance errors during the VFC task would be greater, in both groups when the number of fingers employed in the task increased. We expected that the finger-force output would be adversely affected by the increased degrees of freedom that need to be controlled by the CNS. At the kinetic level, the index (I) finger pressing condition has one variable; index normal force [ $F_{i}^{n}$ ], with one constraint, [ $F_{i}^{n}=F_{\text {const }}^{\text {press }}$ ], where $F_{\text {const }}^{\text {press }}$ is the target constant force for the index finger pressing. Thus with one finger, there is no kinetic redundancy in the system. With similar joint configurations, the following finger conditions (IM, IMR, and IMRL) have consecutively two, three and four variables and the same constraint (the target of constant force), creating an increased number of kinetic redundancy. Unexpectedly, the results of this study did not show effects of kinetic redundancy in the finger force control in either group. Paradoxically, as more fingers were added to the same motor task, the performance error (RMSe) tended to decrease in children with DCD while TD children showed similar performance errors across all the finger conditions $(\sim 3 \%)$. We interpret these results to mean that the typically developing 10-year-old may have developed the ability to synergically solve the problem of reducing the number of joint/muscle-level degrees of freedom in order to achieve a common motor task. On the other hand, children with DCD have not solved the problem. For example, with the one finger (I), the RMSe for children with DCD was almost three times greater than their TD cohorts $(8.5 \%$ compared to $2.9 \%$ for TD, or a difference of $5.6 \%)$. But using multiple fingers (IMRL), decreased this difference (5.6\% compared to $2.9 \%$ or a difference of $2.7 \%$ ). One possible explanation for this result may be that children with DCD benefited from increased sensory information (tactile and proprioceptive) available in the IMRL task. In fact, using more fingers increased the contact surface area between the fingers and the force sensors as well as the number of joints and muscles actively involved in the task. The results for the children with DCD raise an important question about the contrasting effects of motor redundancy and motor-sensory abundance. Clearly for the children with DCD the motor-sensory abundance (increased tactile and proprioceptive information) improved their performance and may offer an important insight into underlying mechanisms of DCD. Future studies are needed to confirm this speculation. 
Although learning and/or adaptation were not a focus of this current study, the children had performed twelve consecutive trials within a condition. We, therefore, used a regression analysis to check for the effect of trial repetition. We expected that over twelve trials, a certain level of improvement in the task would be seen in both groups. The results showed no effect of practice in either group for all finger conditions. We also examined inter-trial variability and found that children with DCD as compared with their TD peers were significantly less consistent over the trials. This finding indicates an absence of a constant visual force control strategy used by children with DCD over twelve trials of procedural practice.

\section{CONCLUSION}

This study aimed to investigate finger independency and visual force control in children with DCD. Our results from the MVF and VFC tasks allowed us to detect that children with DCD as compared with TD children may (a) produce similar levels of maximum finger force; (b) present less finger independency; (c) have similar finger-force sharing patterns; (d) show larger performance errors, and (e) lower inter-trial consistency. Although these preliminary findings cannot be generalized due to the small sample size, we believe that our methodological approach yield a reasonable amount of data and therefore provide reliable measurement of motor function in the individuals studied. Based on our results, we speculate that the impairments in manipulative skills in children with DCD may not related to their strength capability but possibly to their increased level of inter-finger dependency. Although the small group of examined children were able to visually control their force finger at $20 \%$ of their MVF, the greater performance errors found in the group of children with DCD may reflect their inability to well integrate feedback and feedforward strategies.

Additionally, we found that the group of children with DCD had no difficulties in reducing the number of joint/muscle-level degrees of freedom in order to achieve a common motor task. Our findings also suggest that while the group of children with DCD had difficulties in regulating multiple task-level kinetic variables independently, ${ }^{16}$ they do no not appear to be differentially impaired when the number of joint/muscle-level degrees of freedom was increased in the same task.

\section{REFERENCES}

1. Bernstein NA. The co-ordination and regulation of movements. Oxford: Pergamon Pres. 1967.

2. Turvey MT. Coordination. Am Psychol 1990; 45: 938.

3. Latash ML, Shim JK, Zatsiorsky VM. Is there a timing synergy during multifinger production of quick force pulses? Psychopharmacology (Berl) 2004; 177: 217-23.

4. Schieber MH, Poliakov AV. Partial inactivation of the primary motor cortex hand area: effects on individuated finger movements. J Neurosci 1998; 18: 9038-

54.

\begin{tabular}{l|l|l|l|}
\hline Oliveira & 2015 & VOL. 9 & N.2
\end{tabular}


5. Schieber MH, Santello M. Hand function: peripheral and central constraints on performance. J Appl Physiol 2004; 96: 2293-300.

6. Shim JK, Latash ML, Zatsiorsky VM. Prehension synergies in three dimensions. J Neurophysiol 2005; 93: 766-76.

7. Zatsiorsky V, Gao F, Latash M. Prehension synergies: effects of object geometry and prescribed torques. Exp Brain Res 2003; 148: 77-87.

8. Geuze RH. Motor impairment in DCD and activities of daily living. In: Sugden DA, Chambers M, editors. Children with Developmental Coordination Disorder. London: Whurr Publishers; 2005.

9. Polatajko HJ, Macnab JJ, Anstett B, Malloy - Miller T, Murphy K, Noh S. A clinical trial of the process-oriented treatment approach for children with developmental co-ordination disorder. Dev Med Child Neurology 1995; 37: 3109.

10. Lundy-Ekman L, Ivry R, Keele S, Woollacott M. Timing and force control deficits in clumsy children. J Cog Neurosci 1991; 3: 367-76.

11. Piek JP, Skinner RA. Timing and force control during a sequential tapping task in children with and without motor coordination problems. J Int Neuropsychol Soc 1999; 5: 320-9.

12. Pitcher TM, Piek JP, Barrett NC. Timing and force control in boys with attention deficit hyperactivity disorder: subtype differences and the effect of comorbid developmental coordination disorder. Hum Mov Sci 2002; 21: 919-45.

13. Hill EL, Wing AM. Developmental disorders and the use of grip force to compensate for inertial forces during voluntary movement. In: Connolly KJ, editor. The Psychobiology of the Hand. London: Mac Keith Press; 1998. p. 199212.

14. Hill EL, Wing AM. Coordination of grip force and load force in developmental coordination disorder: A case study. Neurocase 1999; 5: 537-44.

15. Pereira HS, Landgren M, Gillberg C, Forssberg H. Parametric control of fingertip forces during precision grip lifts in children with DCD (developmental coordination disorder) and DAMP (deficits in attention motor control and perception). Neuropsychologia 2001; 39: 478-88.

16. Oliveira MA, Shim JK, Loss JF, Petersen RDS, Clark JE. Effect of kinetic redundancy on hand digit control in children with DCD. Neurosci Lett 2006; 410: 42-6.

17. Häger-Ross C, Schieber MH. Quantifying the independence of human finger movements: comparisons of digits, hands, and movement frequencies. J Neurosci 2000; 20: 8542-50.

18. Li Z-M, Latash ML, Zatsiorsky VM. Force sharing among fingers as a model of the redundancy problem. Exp Brain Res 1998; 119: 276-86. 
19. Schieber MH, Hibbard LS. How somatotopic is the motor cortex hand area? Science 1993; 261: 489-92.

20. Shim JK, Oliveira MA, Hsu J, Huang J, Park J, Clark JE. Hand digit control in children: age-related changes in hand digit force interactions during maximum flexion and extension force production tasks. Exp Brain Res 2006; 176: 374-86. 21. Shinohara M, Li S, Kang N, Zatsiorsky VM, Latash ML. Effects of age and gender on finger coordination in MVC and submaximal force-matching tasks. J Appl Physiol 2003; 94: 259-70.

22. Shinohara M, Latash ML, Zatsiorsky VM. Age effects on force produced by intrinsic and extrinsic hand muscles and finger interaction during MVC tasks. J Appl Physiol 2003; 95: 1361-9.

23. Smits-Engelsman BCM, Westenberg Y, Duysens J. Development of isometric force and force control in children. Cogn Brain Res 2003; 17: 68-74.

24. Ameratunga D, Johnston L, Burns Y. Goal - directed upper limb movements by children with and without DCD: a window into perceptuo-motor dysfunction? Physiother Res Int 2004; 9: 1-12.

25. Van Waelvelde H, De Weerdt W, De Cock P, Janssens L, Feys H, Engelsman BCMS. Parameterization of movement execution in children with developmental coordination disorder. Brain Cogn 2006; 60: 20-31.

26. Shim JK, Latash ML, Zatsiorsky VM. Prehension synergies: trial-to-trial variability and principle of superposition during static prehension in three dimensions. J Neurophysiol 2005; 93: 3649-58.

27. Winter DA. Biomechanics and motor control of human movement. Wiley: New York. 2009.

28. Zatsiorsky VM, Li Z-M, Latash ML. Enslaving effects in multi-finger force production. Exp Brain Res 2000; 131: 187-95.

29. Potter NL, Kent RD, Lindstrom MJ, Lazarus J-AC. Power and precision grip force control in three-to-five-year-old children: velocity control precedes amplitude control in development. Exp Brain Res 2006; 172: 246-60.

30. Leijnse J, Walbeehm ET, Sonneveld GJ, Hovius SER, Kauer JMG. Connections between the tendons of the musculus flexor digitorum profundus involving the synovial sheaths in the carpal tunnel. Acta Anat (Basel) 1997; 160: 112-22.

31. von Schroeder HP, Botte MJ, Gellman H. Anatomy of the juncturae tendinum of the hand. J Hand Surg [Am] 1990; 15: 595-602.

32. von Schroeder HP, Botte MJ. Anatomy and functional significance of the long extensors to the fingers and thumb. Clin Orthop Relat Res 2001; 383: 74-83.

33. Hay L, Redon C. The control of goal-directed movements in children: Role of proprioceptive muscle afferents. Hum Mov Sci 1997; 16: 433-51. 
34. Blank R, Heizer W, Von Voß H. Externally guided control of static grip forces by visual feedback-age and task effects in 3-6-year old children and in adults. Neurosci Lett 1999; 271: 41-4.

35. Blank R, Heizer W, Von Voss H. Development of externally guided grip force modulation in man. Neurosci Lett 2000; 286: 187-90.

36. Deutsch KM, Newell KM. Age differences in noise and variability of isometric force production. J Exp Child Psychol 2001; 80: 392-408.

37. Deutsch KM, Newell KM. Children's coordination of force output in a pinch grip task. Dev Psychobiol 2002; 41: 253-64.

38. Deutsch KM, Newell KM. Deterministic and stochastic processes in children's isometric force variability. Dev Psychobiol 2003; 43: 335-45.

39. Lazarus J-AC, Whitall J, Franks CA. Isometric force regulation in children. J Exp Child Psych 1995; 60: 245-60.

40. Smits-Engelsman BCM, Sugden D, Duysens J. Developmental trends in speed accuracy trade-off in 6-10-year-old children performing rapid reciprocal and discrete aiming movements. Hum Mov Sci 2006; 25: 37-49.

41. Santello M, Soechting JF. Force synergies for multifingered grasping. Exp Brain Res 2000; 133: 457-67.

42. Shim JK, Latash ML, Zatsiorsky VM. The human central nervous system needs time to organize task-specific covariation of finger forces. Neurosci Lett 2003; 353: 72-4.

43. Shim JK, Latash ML, Zatsiorsky VM. Prehension synergies: trial-to-trial variability and hierarchical organization of stable performance. Exp Brain Res 2003; 152: 173-84.

44. Shim JK, Lay BS, Zatsiorsky VM, Latash ML. Age-related changes in finger coordination in static prehension tasks. J Appl Physiol 2004; 97: 213-24.

Citation: Marcio A. Oliveira. Preliminary findings of finger independency and visual force control in children with DCD. BJMB. 2015: 9(2): 1-19.

Editor: Joao A. C. Barros, California State University Fullerton, Fullerton, CA, USA.

Copyright: @ 2015 Marcio A. Oliveira and BJMB. This is an open-access article distributed under the terms of the Creative Commons Attribution-NonCommercial-NoDerivatives 4.0 International License which permits unrestricted use, distribution, and reproduction in any medium, provided the original author and source are credited.

Competing interests: The authors have declared that no competing interests exist.

Download: http://socibracom.com/bjmb/index.php/bjmb/issue/view/25 